\title{
Plasticity of Schwann cells and pericytes in response to islet injury in mice
}

\author{
Shiue-Cheng Tang • Yu-Chen Chiu • Chia-Tung Hsu • \\ Shih-Jung Peng • Ya-Yuan Fu
}

Received: 3 May 2013 / Accepted: 11 June 2013 / Published online: 26 June 2013

(C) Springer-Verlag Berlin Heidelberg 2013

\begin{abstract}
Aims/hypothesis Islet Schwann (glial) cells and pericytes are the microorgan's accessory cells positioned at the external and internal boundaries facing the exocrine pancreas and endothelium, respectively, adjacent to the endocrine cells. Plasticity of glial cells and pericytes is shown in the glial scar formation after injury to the central nervous system. It remains unclear whether similar reactive cellular responses occur in insulitis. We applied three-dimensional (3D) histology to perform qualitative and quantitative analyses of the islet Schwann cell network and pericytes in normal, streptozotocin-injected (positive control of gliosis) and NOD mouse models.

Methods Vessel painting paired with immunostaining of mouse pancreatic tissue was used to reveal the islet Schwann cells and pericytes and their association with vasculature. Transparent islet specimens were prepared by optical clearing to facilitate 3D confocal microscopy for panoramic visualisation of the tissue networks.
\end{abstract}

Electronic supplementary material The online version of this article (doi:10.1007/s00125-013-2977-y) contains peer-reviewed but unedited supplementary material, which is available to authorised users.

S.-C. Tang • Y.-C. Chiu • C.-T. Hsu • S.-J. Peng • Y.-Y. Fu

Connectomics Research Center, National Tsing Hua University,

Hsinchu, Taiwan

S.-C. Tang $(\varangle) \cdot$ S.-J. Peng $\cdot$ Y.-Y. Fu

Institute of Biotechnology, National Tsing Hua University,

101, Sec. 2, Kuang Fu Rd, Hsinchu 30013, Taiwan

e-mail: sctang@life.nthu.edu.tw

S.-C. Tang

Department of Medical Science, National Tsing Hua University,

Hsinchu, Taiwan

Present address:

Y.-Y. Fu

Division of Gastroenterology and Hepatology, Johns Hopkins

University School of Medicine, Baltimore, MD, USA
Results In-depth microscopy showed that the islet Schwann cell network extends from the peri-islet domain into the core. One week after streptozotocin injection, we observed intraislet perivascular gliosis and an increase in pericyte density. In early/moderate insulitis in the NOD mice, perilesional gliosis occurred at the front of the lymphocytic infiltration with atypical islet Schwann cell morphologies, including excessive branching and perivascular gliosis. Meanwhile, pericytes aggregated on the walls of the feeding arteriole at the peri- and intralesional domains with a marked increase in surface marker density.

Conclusions/interpretation The reactive cellular responses demonstrate plasticity and suggest a stop-gap mechanism consisting of the Schwann cells and pericytes in association with the islet lesion and vasculature when injury occurs.

Keywords Gliosis · Insulitis · Islet · NOD mouse · Pericyte · Plasticity $\cdot$ Schwann cell $\cdot$ Streptozotocin

$\begin{array}{ll}\text { Abbreviations } \\ \text { 2D } & \text { Two-dimensional } \\ \text { 3D } & \text { Three-dimensional } \\ \text { CNS } & \text { Central nervous system } \\ \text { GFAP } & \text { Glial fibrillary acidic protein } \\ \text { NG2 } & \text { Neuron-glial antigen 2 } \\ \text { STZ } & \text { Streptozotocin }\end{array}$

\section{Introduction}

The endocrine islets of Langerhans are the regional neurovascular hubs in the pancreas and constantly receive signals from the circulation and nerves in response to physiological cues [1]. Associated with the rich nerve and blood supplies, islets are sheathed in a dense network of the processes of Schwann cells (the glial cells of the peripheral 
nervous system) $[2,3]$ and are positioned with pericytes along the capillaries $[4,5]$. However, due to the dispersed nature of the neural and vascular tissues, characterisation of the islet Schwann cells and pericytes in health and disease has been difficult, even in animals.

Notably, in the central nervous system (CNS), gliosis and glial scar formation, consisting of the glial cells and pericytes surrounding the lesion, are the hallmarks of Alzheimer's disease, stroke and traumatic injury [6-8]. In the enteric nervous system, increases in the expression of glial fibrillary acidic protein (GFAP) and proliferation of glial cells have also been reported in bowel inflammation such as ulcerative colitis [9-12]. As to the 'neuroendocrine' islet, it remains unclear whether similar reactive cellular responses occur in islet injuries, such as insulitis, in the progression of type 1 diabetes.

Using NOD mice, which have a condition resembling type 1 diabetes with insulitis caused by lymphocytic infiltration, Winer et al discovered that the autoimmune target tissues include both pancreatic beta cells and Schwann cells [13]. They found that the peri-islet lymphocytic accumulation in insulitis creates disturbances and breaches of the glial sheath prior to the autoimmune attack on the beta cells [14-16]. Interestingly, in contrast to the destruction of Schwann cells, reactive gliosis was also reported in streptozotocin (STZ)-injected mice [17]. The variation in islet glial-cell content in animal models of diabetes highlights Schwann cells as a dynamic cellular factor involved in islet injury and inflammation.

In rodent models of obesity and hypertension, histological changes in islet pericytes, including hypertrophy and an increase in staining density of the surface marker neuronglial antigen 2 (NG2), have been observed [4, 5, 18, 19], suggesting pericytes' morphological plasticity in response to the disturbances of the circulation. In the progression of type 1 diabetes, although the role of pericytes is not understood, vascular swelling and leakage have been imaged in insulitis and used as an indicator of islet inflammation in NOD mice $[20,21]$. Whether or not the vascular damage induces remodelling of pericytes is the topic for investigation in this research.

To elucidate the activity of Schwann cells and pericytes in islet injury, we prepared transparent islet specimens by optical clearing [22-25] and combined vessel painting (perfusion of fluorescent lectin) with three-dimensional (3D) histology [26-32]. This allowed the identification of the spatial features of the $\mathrm{GFAP}^{+}$Schwann cells and $\mathrm{NG}^{+}$pericytes (note: although named as a neuron-' glial' antigen, the NG2 cell-surface chondroitin sulfate proteoglycan is highly expressed in the pancreatic/islet pericytes and has been used as a histological marker $[5,33])$ and their association with the islet lesion and vasculature [13, 17, 34-36]. Qualitative and quantitative analyses of the islet injury-induced gliosis and pericyte remodelling around the feeding arteriole, and their pathophysiological implications, are presented and discussed in this report.

\section{Methods}

Animals Pancreases harvested from female BALB/c mice (BioLASCO, Taipei, Taiwan) were used to acquire the images of normal islets. Pancreases harvested from streptozotocin (Sigma, St Louis, MO, USA)-injected female BALB/c mice (age 7-8 weeks; single i.p. injection, $180 \mu \mathrm{g} / \mathrm{g}$ body weight; killed at one week after the injection, positive control of gliosis [17]) and female NOD mice (age 7-8, 11-14 and 20-22 weeks; National Laboratory Animal Center, Taipei, Taiwan) were used to acquire images of different scales of insulitis. Schwann cell destruction in severe insulitis was used as the negative control of GFAP staining.

Islet injury induced by STZ injection was confirmed by hyperglycaemia $(6 \mathrm{~h}$ fasting glucose concentration $>8.3$ $\mathrm{mmol} / \mathrm{l}$ ) measured on day 3 (from the tail tip with an AccuCheck Performa glucometer; Roche, Indianapolis, IN, USA) and day 7 before mice were killed (six out of eight STZinjected mice developed hyperglycaemia; only the mice with hyperglycaemia were used in microscopy and analysis).

The glucose levels of the untreated BALB/c and NOD mice in early and moderate insulitis were within the normal range (3.9-6.7 $\mathrm{mmol} / \mathrm{l})$. The young NOD mice were used to study the islet lesions and perilesional tissue remodelling induced by autoimmune progression. The aged NOD mice with severe insulitis and hyperglycaemia were used as a negative control to reveal the destruction of the Schwann cell network, as previously shown by Winer et al [13]. Overall, 44, 30 and 36 image stacks derived from six normal BALB/c mice, six STZ-injected BALB/c mice and eight NOD mice (three at age 7-8 weeks, three at age 11-14 weeks and two at age 20-22 weeks), respectively, were used to generate representative images. The National Tsing Hua University Institutional Animal Care and Use Committee approved all procedures involving mice.

Tissue labelling Vessel painting [30, 34, 35] was performed by cardiac perfusion of the lectin (wheat germ agglutinin)Alexa Fluor 488 conjugate $(30 \mu \mathrm{g} / \mathrm{g}$ body weight; Cat. No. W11261; Invitrogen, Carlsbad, CA, USA) (pseudo-coloured red was assigned to the signals of blood vessels in the images) followed by $4 \%$ paraformaldehyde perfusion fixation. After this, pancreases were harvested and the vibratome sections of the tissue $(\sim 400 \mu \mathrm{m})$ were post-fixed in $4 \%$ paraformaldehyde solution for $1 \mathrm{~h}$ at $25^{\circ} \mathrm{C}$. The fixed tissues were then immersed in $2 \%$ Triton X-100 solution for $2 \mathrm{~h}$ at $25^{\circ} \mathrm{C}$ for permeabilisation. 
The primary antibodies used to reveal the islet Schwann cells and pericytes were a polyclonal rabbit anti-GFAP antibody (DAKO, Z0334; Carpinteria, CA, USA) and a rabbit anti-NG2 antibody (AB5320; Millipore, Billerica, MA, USA), respectively. Before applying the antibody, the tissue was rinsed in PBS. This was followed by a blocking step, in which the tissue was incubated with a blocking buffer $(2 \%$ Triton X-100, 10\% normal goat serum and $0.02 \%$ sodium azide in PBS). The primary antibody was then diluted with a dilution buffer $(1: 50 ; 0.25 \%$ Triton X-100, $1 \%$ normal goat serum and $0.02 \%$ sodium azide in PBS) to replace the blocking buffer and incubated for 1 day at $15^{\circ} \mathrm{C}$.

The Alexa Fluor 647-conjugated goat anti-rabbit secondary antibody (1:200; Invitrogen) was used to reveal the immunostained structure. Then nuclear staining by propidium iodide $(50 \mu \mathrm{g} / \mathrm{ml}$; Invitrogen) was performed at room temperature for $1 \mathrm{~h}$. The labelled specimens were then immersed in the optical-clearing solution FocusClear (CelExplorer, Hsinchu, Taiwan) overnight before being imaged via confocal microscopy [37]).

Confocal microscopy A Zeiss LSM 510 Meta confocal microscope (Carl Zeiss, Jena, Germany) equipped with the objectives of $20 \times$ (optical section: $5 \mu \mathrm{m}$; Z-axis increment: $2.5 \mu \mathrm{m}$ ) 'Fluar' lenses and $40 \times$ (optical section: $3 \mu \mathrm{m}$; Z-axis increment: $1.5 \mu \mathrm{m}) \mathrm{LD}$ 'C-Apochromat' water immersion lenses (working distance: $620 \mu \mathrm{m}$ ) was used to acquire the images. We used transmitted light microscopy in combination with confocal microscopy to examine the specimens. Each micrograph consisted of 1,024 (X) $\times 1,024(\mathrm{Y})$ pixels. The laser-scanning process was operated under the multitrack scanning mode to sequentially acquire signals in multiple channels. The Alexa Fluor 647-labelled structures were excited at $633 \mathrm{~nm}$ and the fluorescence was collected by the $650-710 \mathrm{~nm}$ band-pass filter. The propidium iodide-labelled nuclei were excited at $543 \mathrm{~nm}$ and the signals were collected by the $560-615 \mathrm{~nm}$ band-pass filter. The lectin-Alexa Fluor 488-labelled blood vessels were excited at $488 \mathrm{~nm}$ and the fluorescence was collected by the $500-550 \mathrm{~nm}$ band-pass filter.

Image projection and analysis LSM 510 software (Carl Zeiss) and Avizo 6.2 image reconstruction software (VSG, Burlington, MA, USA) were used for projection, signal segmentation and analysis of the confocal images. Figures $1 \mathrm{~b}-\mathrm{f}, 2 \mathrm{~b}, \mathrm{c}, 3 \mathrm{c}, \mathrm{d}, 4 \mathrm{a}, \mathrm{b}, 5 \mathrm{~b}, \mathrm{c}, \mathrm{f}-\mathrm{l}$ and $6 \mathrm{a}, \mathrm{b}, \mathrm{e}$ and Electronic supplementary material (ESM) Figs $1 b-d, 2 b-d$ and 3 were derived from the projection module of the LSM 510 software. In ESM Videos, image stacks were recorded using the 'Movie Maker' function of Avizo with the increase in display time in association with the depth of the optical section. The $360^{\circ}$ presentations in ESM Videos 1 and 2 were derived from the Panorama function of the LSM 510 software. Feature extraction and image segmentation for quantitative analysis were performed by the 'Label Field' function of Avizo to collect the voxels of islets and their associated Schwann cells, pericytes and blood vessels. In gliosis analysis, the voxels of the $\mathrm{GFAP}^{+}$fibres that follow the curvature of the islet surface were used to define the Schwann cell sheath and the islet mantle (3 or 4 nuclei away from the islet surface; use of the 'Shrink' function of Avizo to geometrically follow the curvature of the surface). In the 'Label Field' calculation, the voxels of the islet core are equal to the overall islet voxels minus the voxels of the mantle.

Quantification of pericytes in the normal pancreas (Fig. 2d) and the STZ-treated islets (Fig. 4c) was performed by counting the pericytes in the segmented volume of interest (endocrine islet or exocrine acini) for estimation of the cell densities. While counting the pericytes, both the NG2 and nuclear signals were used to define a pericyte - an NG2 immunoreactive cell body with at least two processes contacting the blood vessels. The 'Label Field' function of Avizo was used to label and measure the endocrine and exocrine tissue volumes. For quantification of the pericyte remodelling on the feeding arteriole (Fig. 6f), we first chose and segmented the voxels of the arteriole, such as the blood vessels in the squashed circle in Fig. 6e. We then calculated the NG2 staining density by dividing the overall NG2 signals in the selected area by the voxels of the arteriole in the 3D image stack.

Four normal and four STZ-injected BALB/c mice (12 image stacks each) were used to estimate the increase in GFAP signals in the islets after STZ injection. Three normal and four STZ-injected BALB/c mice were used to estimate the pericyte density in the normal pancreas (13 and 12 image stacks of endocrine islets and exocrine acini, respectively) and in the STZ-treated islets (15 image stacks), respectively. Three normal BALB/c mice ( 8 image stacks) and five NOD mice in early/moderate insulitis (12 image stacks) were used to estimate the NG2 staining density in the blood vessels. Signal or pericyte densities derived from different image stacks of the same mouse were first normalised and then averaged over the other mice in the same group.

Statistics The quantitative values are presented as means \pm SD. Statistical differences were determined by the unpaired Student's $t$ test. Differences between groups were considered statistically significant when $p<0.05$.

\section{Results}

In-depth characterisation of the peri- and intra-islet Schwann cell network Owing to the dispersed nature of the cellular processes, the standard microtome-based tissue 
a

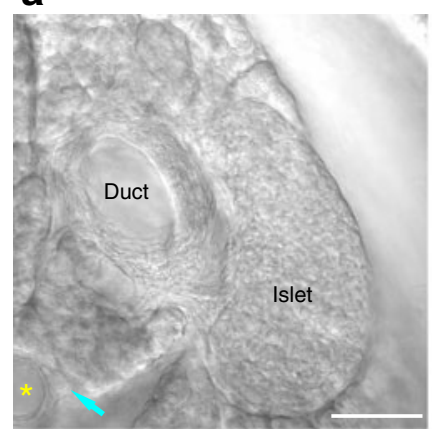

d

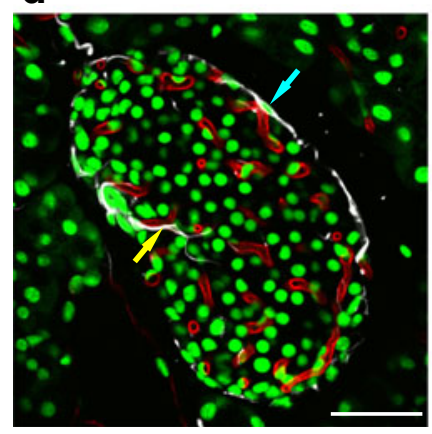

b

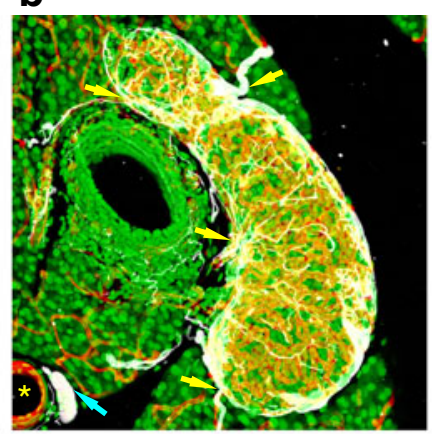

e

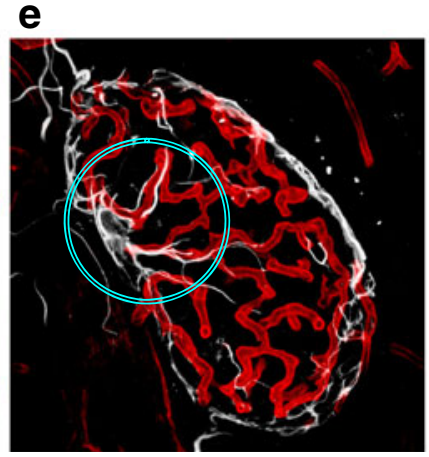

C

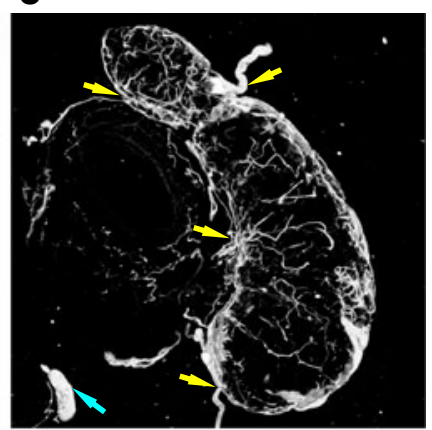

f

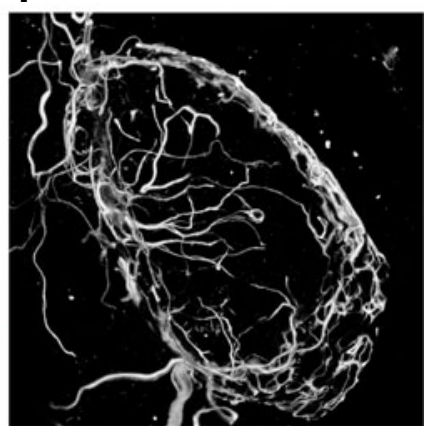

Fig. 1 In-depth characterisation of the islet Schwann cell network. (a-c) Gross view of the Schwann cell network. (a) Transmitted light image of the optically cleared pancreatic tissue (normal BALB/c). The islet, duct and other tissue components, such as the arteriole (asterisk) and ganglion (blue arrow) can be identified. Scale bar: $100 \mu \mathrm{m}$. (b,c) Projections of the GFAP $^{+}$Schwann cell network in the pancreatic tissue; white, GFAP; red, blood vessels; green, nuclei; projection depth, $60 \mu \mathrm{m}$. The morphologies show that the Schwann cell plexus has multiple entry points (yellow arrows) from the exocrine domain into the islet mantle (condensed fibre sheath) and core (slender fibres but marked presence). ESM Video 1 shows in-depth observation and $360^{\circ}$ projection of the Schwann cell

analysis cannot provide an integral view of the islet Schwann cell network. To increase the imaging depth, we prepared transparent islet specimens by optical clearing [28], which allowed us to use transmitted light microscopy to identify the major pancreatic components (the endocrine islet, pancreatic duct and exocrine acini) and visualise the peri- and intra-islet Schwann cell fibres via deep-tissue confocal microscopy (Fig. 1a-c and ESM Video 1 [first half]).

Three features of the Schwann cell network were seen in the gross image of islets in the pancreas. First, we observed minimal, if any, Schwann cell fibres associated with the acini in comparison with the condensed islet Schwann cell sheath and the slender fibres around the duct. Second, the Schwann cell plexus entered from the exocrine domain with multiple entry points into the islet mantle, forming a condensed fibre sheath. Third, we made an unexpected discovery, that the intra-islet Schwann cell fibres extended from the peri-islet domain into the core.

Using the transparent specimen, we next zoomed in to investigate the intra-islet Schwann cell fibres. Both in-depth network. (d-f) Visualisation of the intra-islet Schwann cell fibres with high definition. (d) Close-up view of a pancreatic islet $(3 \mu \mathrm{m}$ optical section) (white, GFAP; red, blood vessels; green, nuclei); scale bar, $50 \mu \mathrm{m}$. The high-resolution image allows us to identify the Schwann cell body (blue arrow) associated with the GFAP-labelled processes. The yellow arrow indicates the elongation of the Schwann cell processes into the core. (e) Projection of GFAP and capillaries (depth, $21 \mu \mathrm{m}$ ). (f) Projection of GFAP (depth, $60 \mu \mathrm{m}$ ). The extensions of the Schwann cell fibres are, in part, adjacent to the islet microvessels (circle in [e]). The entire image stack is shown in the second half of ESM Video 1

observation and projections (Fig. 1d-f and ESM Video 1 [second half]) confirmed that the Schwann cell plexus sprouted from the sheath with processes extending into the core. In addition, the paths of the Schwann cell extensions were, in part, adjacent to those of the microvessels, implying their intimate association. The elongated Schwann cell processes from the mantle into the core suggested a previously overlooked role of the network, likely that it senses/monitors the intra-islet microenvironment, in addition to sheathing the islet.

To verify the endocrine islet structure, paired immunostaining of glucagon with GFAP was used to reveal the periislet alpha cells and the Schwann cell sheath (ESM Fig. 1). The glucagon-expressing alpha cells were used as a reference to define the mantle of the mouse islet, in which the peri-islet Schwann cell network and alpha cells were in contact with each other and in close proximity, confirming the location of the GFAP immunostaining signals.

$3 D$ Characterisation of islet pericytes Similarly to the Schwann cells, pericytes with their long processes in space 
a
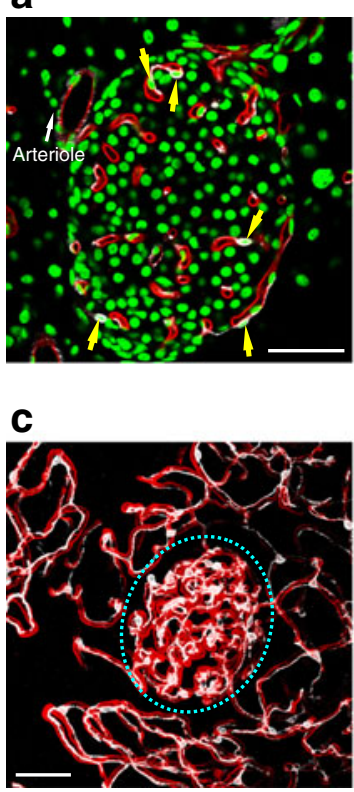

b

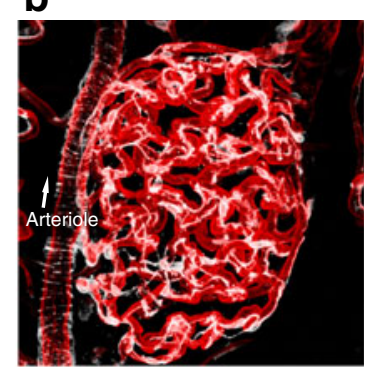

d

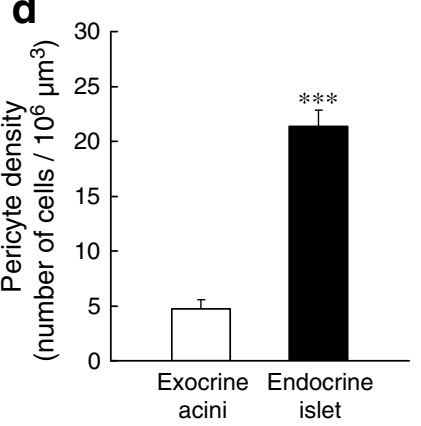

Fig. 2 In-depth characterisation of islet pericytes. $(\mathbf{a}, \mathbf{b})$ Association of pericytes with islet vasculature. Panels (a) and (b) were derived from the same image stack shown in the first part of ESM Video 2. (a) 2D Image of the NG2 (white)-labelled pericytes (normal BALB/c); red, blood vessels; green, nuclei; scale bar, $50 \mu \mathrm{m}$. Yellow arrows indicate the cell bodies of pericytes. The white arrow on the left indicates the direction of an arteriole. (b) Projection of the image stack reveals the $\mathrm{NG}^{+}$processes encircling the arteriole and their elongation on the capillaries in the islet; projection depth, $84 \mu \mathrm{m}$. (c,d) Qualitative and quantitative analyses of the pancreatic/islet pericytes. (c) Distribution of pericytes in the endocrine (circle) and exocrine domains; scale bar, $50 \mu \mathrm{m}$; projection depth, $84 \mu \mathrm{m}$. (d) Quantification shows that the pericyte density is significantly higher in the endocrine islet than in the exocrine acini. Data were derived from normal BALB/c mice $(n=3)$ and presented as mean $\pm \mathrm{SD} .{ }^{* * *} p<0.001 \mathrm{vs}$ exocrine acini

cannot be easily portrayed by standard two-dimensional (2D) microscopy. We used the developed penetrative imaging method with NG2 staining to characterise the 3D features of the pericytes. Figure 2a-c, ESM Fig. 2 and ESM Video 2 present three examples of the immunolabelled pancreatic/islet pericyte population associated with the vasculature (normal $\mathrm{BALB} / \mathrm{c}$ ). As can be seen, the $\mathrm{NG}_{2}{ }^{+}$pericytes consisted of a cell body with a prominent nucleus (yellow arrows in Fig. 2a) and processes extending from the cell body to embrace the abluminal side of the vessel wall. This morphology was consistent with the 2D pericyte images shown in the literature $[5,33]$.

Importantly, through in-depth projection of pericytes with blood vessels, two additional features of the pancreatic/islet pericytes were seen. First, we observed the $\mathrm{NG}^{+}$ processes/fibres encircling the arterioles (white arrows in Fig. 2a,b) in comparison with the extended $\mathrm{NG}^{+}$processes on the capillaries. Second, quantification of pericytes showed that the pericyte density was approximately fourfold

higher in the endocrine islet than in the exocrine acini (Fig. 2c,d) (note: we quantified the pericytes as the $\mathrm{NG}^{+}$ cells with at least two processes extending from the cell body in association with the blood vessels). The higher pericyte density in the islet reflected its rich vascularisation.

Plasticity of Schwann cells and pericytes in response to STZinduced islet injury Teitelman et al reported that STZinduced islet injury stimulates neurotrophin expression and reactive gliosis of the Schwann cells [17]. Because STZ injection also causes islet microvascular damage [38-41], we sought to investigate the remodelling of Schwann cells and pericytes around the blood vessels after STZ injection (Fig. 3).

Figures $3 \mathrm{a}-\mathrm{d}$ and ESM Video 3 show a middle-sized islet $(\sim 150 \mu \mathrm{m})$ with reactive gliosis one week after the STZ injection. Merged projection of GFAP and capillaries revealed intra-islet perivascular gliosis, highlighted by the abundant and dispersed Schwann cell fibres contacting and docking on the capillary walls from different directions in the islet core. This is different from the elongation of the fibres following the paths of the capillaries seen in the normal islet (Fig. 1d-f). The induced intra-islet perivascular gliosis suggests that the Schwann cells not only form a sheath to separate the islet from acini but also sense the change inside the islet in response to injury.

Quantification of the GFAP signals showed that in the islet core, the density of the $\mathrm{GFAP}^{+}$Schwann cell fibres was significantly higher than the normal density following the STZ injection (3.9-fold increase, Fig. 3e). In the mantle, however, because the islets had already been sheathed with a dense network of the processes of Schwann cells, there was no statistical difference between the normal and diseased condition.

For the pericytes, we used high-resolution 3D microscopy to detect the changes following STZ injection. Morphological analysis (Fig. 4) revealed atypical lateral spreading of the pericyte processes (yellow arrows in Fig. 4b) compared with the slender, longitudinal extensions of the normal processes on the capillary walls (Fig. 4a). Quantitative analysis (Fig. 4c) showed a modest $66 \%$ increase in pericyte density in the STZ-treated islet compared with the untreated control.

Lymphocytic infiltration induces localised gliosis in early and moderate insulitis in NOD mice Unlike the generalised intra-islet injury induced by STZ injection, the lymphocytic accumulation in early insulitis in NOD mice caused localised islet injury (Fig. 5). The hallmark of the inflammatory response was the accumulation of lymphocytes between the duct and the islet [42]. The lymphocytic invasion not only damaged the vasculature but also induced gliosis surrounding the influenced area (Fig. 5a-c). 
a

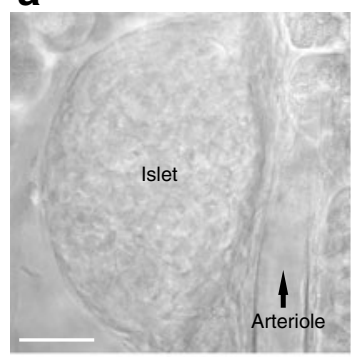

C

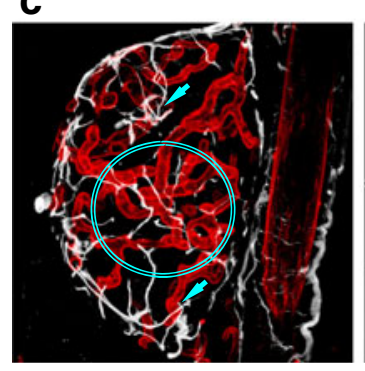

b

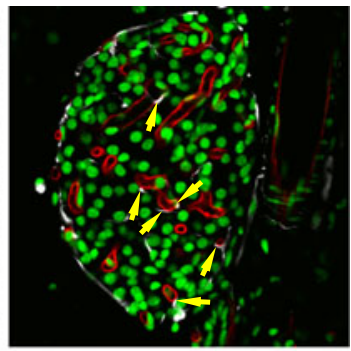

d

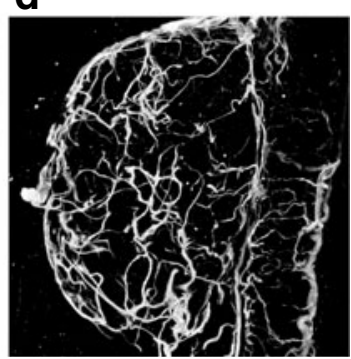

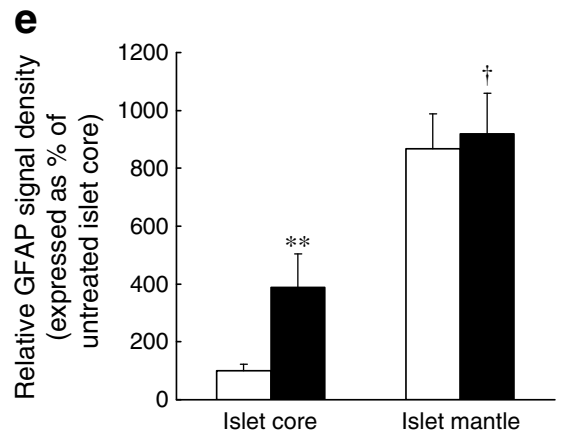

Fig. 3 STZ injection induces reactive cellular responses of Schwann cells. (a-d) Intra-islet perivascular gliosis induced by STZ injection. Panels (a-d) were derived from the same image stack (ESM Video 3). (a) Transmitted light micrograph; scale bar, $50 \mu \mathrm{m}$. (b) Still image from ESM Video 3; white, GFAP; red, blood vessels; green, nuclei. Yellow arrows indicate the perivascular GFAP ${ }^{+}$fibres. (c) Projection of GFAP and capillaries (depth, $21 \mu \mathrm{m}$ ). (d) Projection of GFAP (depth, $60 \mu \mathrm{m}$ ). Parts (c) and (d) illustrate the connections of the GFAP ${ }^{+}$fibres in space and their perivascular presence (blue arrows and circle). (e) Quantification shows a significant increase in GFAP signals in the islet core after
STZ injection, but not in the mantle; white bar, untreated control; black bar, STZ-treated islet. We quantified the islet glial density by dividing the voxels occupied by the GFAP signals with those of the islets in the acquired 3D images. Specifically, we used the voxel signals of the $\mathrm{GFAP}^{+}$fibres, which followed the curvature of the islet surface, to define the Schwann cell sheath and islet mantle (a span of approximately three or four nuclei from the islet surface). Data were derived from the normal $(n=4)$ and STZ-injected $(n=4)$ mice and presented as means \pm SD. ${ }^{* *} p<0.01$ vs control; ${ }^{\dagger} p=0.30$ vs control
Figure $5 \mathrm{~d}-\mathrm{f}$ shows the close-up view of the localised gliosis in early insulitis. Taking advantage of the transparent specimen, we acquired in-depth images of Schwann cells to demonstrate their close association with the infiltrated lymphocytes (ESM Video 4 [first half]). Zoom-in examination and projections of the Schwann cell processes and capillaries in Fig. 5e,f revealed that the induced gliosis occurred at the front of the infiltrated area against the normal domain (perilesional gliosis) with abundant short extensions sprouting from the Schwann cell sheath to contact the islet capillaries from various directions (perivascular gliosis). This morphology differed from the normal smooth and elongated Schwann cell fibres extending from the mantle into the core (Fig. 1d,f).

In moderate insulitis the perivascular gliosis extended from the mantle to the core together with the advancement of lymphocytic infiltration (Fig. 5g-i). High-resolution images at the infiltration boundary revealed perivascular gliosis with excessive branching of the Schwann cell processes on the capillary walls (Fig. 5i and ESM Video 4 [last part]). ESM Fig. 3 shows a second example of an islet in moderate insulitis, in which perivascular gliosis and the Schwann cell breach were observed simultaneously at different corners of the islet. The breach underlined the breakdown of the Schwann cell network due to the autoimmune attack as reported by Winer et al [13].

In severe insulitis, destruction of the Schwann cell network became prominent in the NOD mice (Fig. $5 \mathrm{j}-1$ ). Under the intensive autoimmune attack, islet destruction was accompanied by vascular damage and dismantling of the Schwann cell network. Nonetheless, prior to the destruction, the transitory and localised gliosis and its association with the lesion indicate the plasticity of the islet Schwann cells and their participation in the inflammatory response in insulitis.

Pericyte aggregation around the feeding arteriole in insulitis in NOD mice In addition to the perilesional gliosis, the lymphocytic infiltration also led to changes of pericytes around the islet feeding arteriole in the NOD mice. Unlike the STZ-induced global change of islet pericytes on the capillaries, lymphocytic infiltration primarily induced localised pericyte aggregation around the feeding arteriole at locations close to the islet pole. This phenomenon occurred while the lymphocytes arrived at the peri-islet domain 


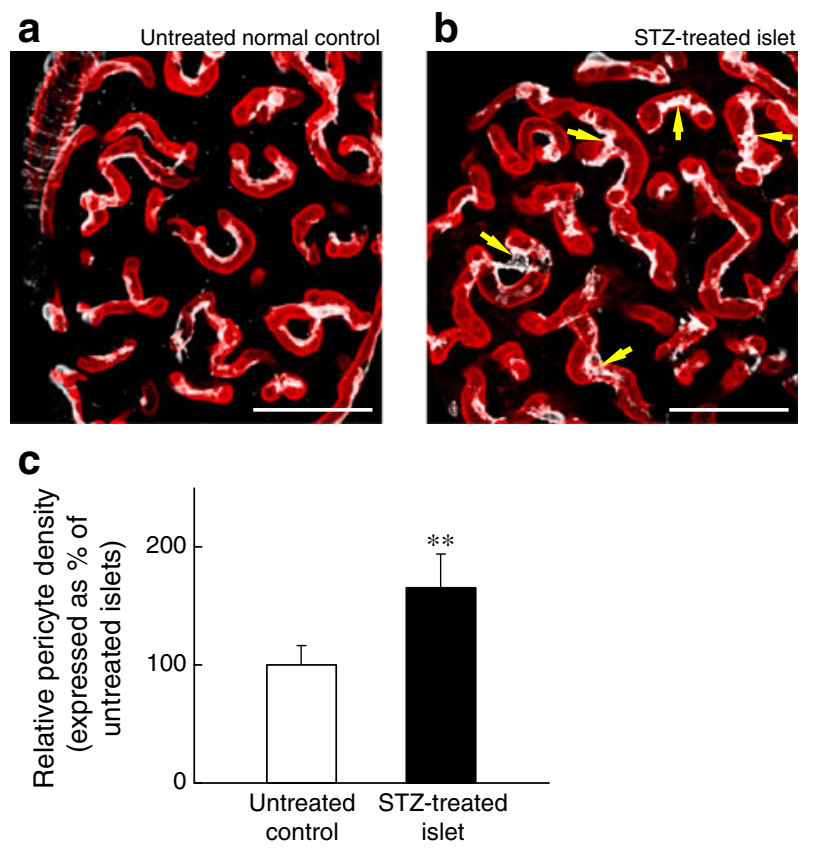

Fig. 4 Morphological and quantitative analyses of pericytes in the STZ-treated islets. (a,b) Projections of the image stack show lateral spreading of the NG2-labelled pericyte processes on the capillary walls of an STZ-treated islet (b) relative to the normal control (a), the latter derived from a subset of Fig. 2b; white, NG2; red, blood vessels. Panels (a) and (b) were projected using the same magnification and dimensions (scale bar, $50 \mu \mathrm{m}$; projection depth, $15 \mu \mathrm{m}$ ). Yellow arrows in (b) indicate the atypical spreading and short protrusions of the pericyte processes. (c) Quantitative analysis shows an increase in pericyte density in the STZ-treated islets compared with the untreated controls. Data were derived from normal $(n=3)$ and STZ-injected $(n=4)$ mice and presented as means $\pm \mathrm{SD} .{ }^{* *} p<0.01$ vs untreated control

(Fig. 6a,b) and persisted as the lymphocytes migrated into the core (Fig. 6c-e). The remodelling of pericytes on the walls of arterioles was distinctly observed in projection. We analysed 12 islets derived from five NOD mice in early/moderate insulitis to confirm this remodelling of pericytes on the walls of arterioles. The marked increase (4.7-fold) in the NG2 staining density on the arteriole was quantified (Fig. 6f) by collecting the signals of the $\mathrm{NG}^{+}$ processes enclosing the blood vessel against those of the slender, perivascular $\mathrm{NG}_{2}^{+}$processes. The morphological and quantitative changes in pericytes on the feeding arteriole, as well as the perilesional and perivascular gliosis of Schwann cells in lymphocytic infiltration, highlight the plasticity of the two cell types in the progression of autoimmune diabetes.

\section{Discussion}

The role of Schwann cells and pericytes in normal islet function is not completely understood, but their changes in response to islet injury indicated that they are not inert bystanders in experimental diabetes. Instead, they reacted to STZ injection and autoimmune progression in mouse models of diabetes. In particular, the Schwann cells responded to lymphocytic infiltration with gliosis occurring at the boundary of the islet lesion in early and moderate insulitis (perilesional gliosis, Fig. 5a-i). Gliosis was also found around the islet capillaries as the lesion progressed from the mantle into the core (perivascular gliosis, Fig. 5d-i). In the meantime, changes in pericytes occurred on the islet feeding arteriole at the peri- and intralesional regions (Fig. 6). Aggregation of pericytes, with their processes enclosing the vessel wall, underlined the pericytes' plasticity. The reactive cellular responses demonstrate the plasticity and suggest a stop-gap mechanism consisting of the Schwann cells and pericytes in association with the islet lesion and vasculature while injury occurs in the autoimmune progression.

Studies on scar tissue in CNS injures have inspired this investigation into the Schwann cells and pericytes in islet injury. The formation of glial scar (including a stromal component derived from pericytes [8]) in CNS injuries is thought to help restore the blood-brain barrier. Here, in the progression of the islet lesion in the NOD mice, the Schwann cells and pericytes also targeted blood vessels in their reactive cellular responses, albeit without forming a scar. Nonetheless, the reactive cellular responses underline the critical role of the islet circulation in its endocrine functions.

In the NOD mice, we demonstrated that perilesional gliosis occurs in early and moderate insulitis before the destruction of the Schwann cells in severe insulitis. Using the same mouse model, Winer and colleagues showed that the accumulation of $\mathrm{T}$ cells around the islet first disrupts the Schwann cell network to create breaches before the attack on the beta cells [13, 43]; however, their study did not identify the phase of gliosis or the formation of the glial-vascular complex in the autoimmune progression. Based on our data shown in Fig. 5 and ESM Video 4, we propose that the Schwann cell disruption includes two phases: first, gliosis, which associates with the lesion and amplifies the antigenic/inflammatory signals (due to the antigen presenting ability of the Schwann cells $[44,45])$ and, second, destruction, as a consequence of the lymphocytic attack.

Although we identified two new cellular responses that occurred in the islet lesion progression, we did not study their interactions. This is because the current tissue labelling and confocal imaging designs were limited to three fluorescence channels, which we felt confident avoided signal 

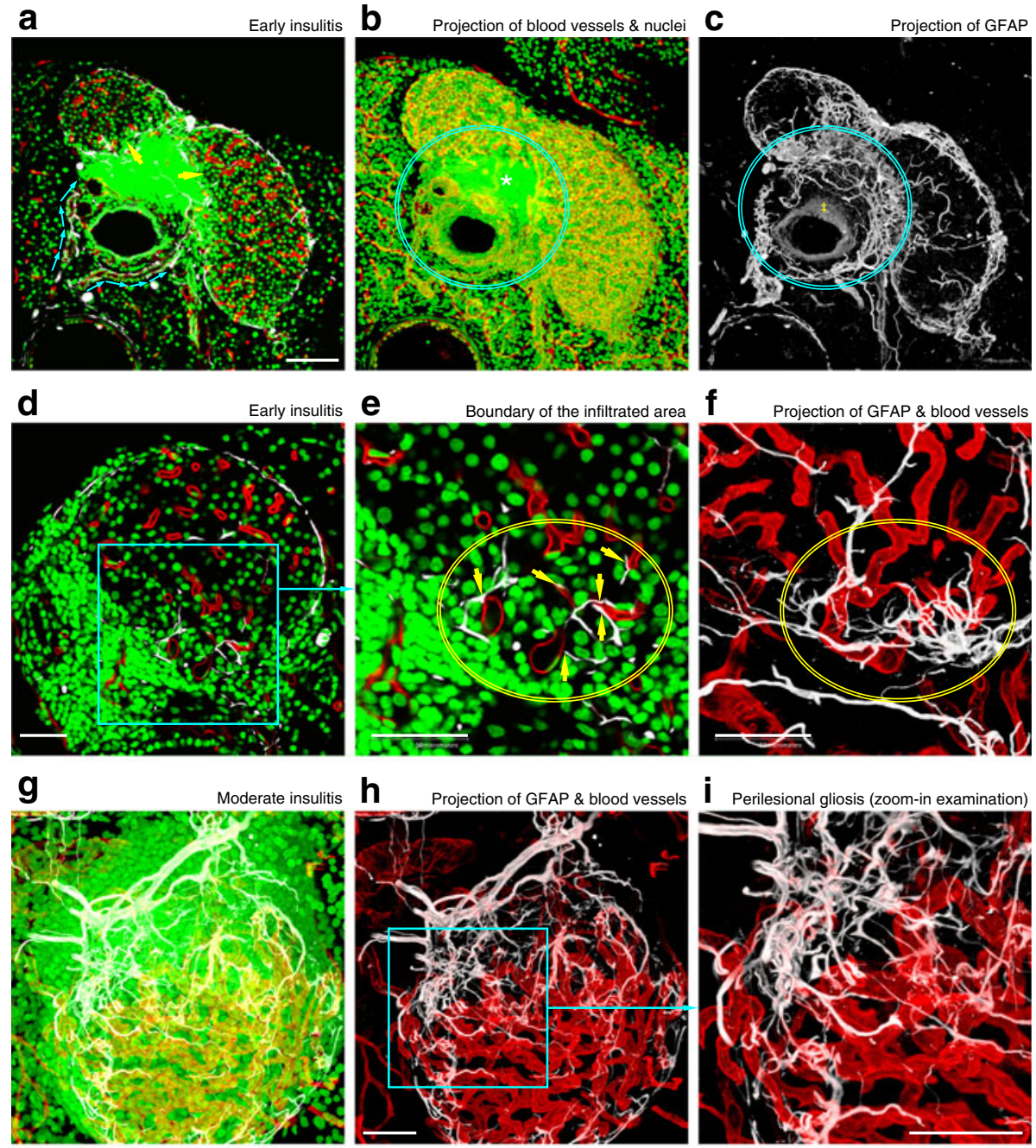

h

Projection of GFAP \& blood vessels
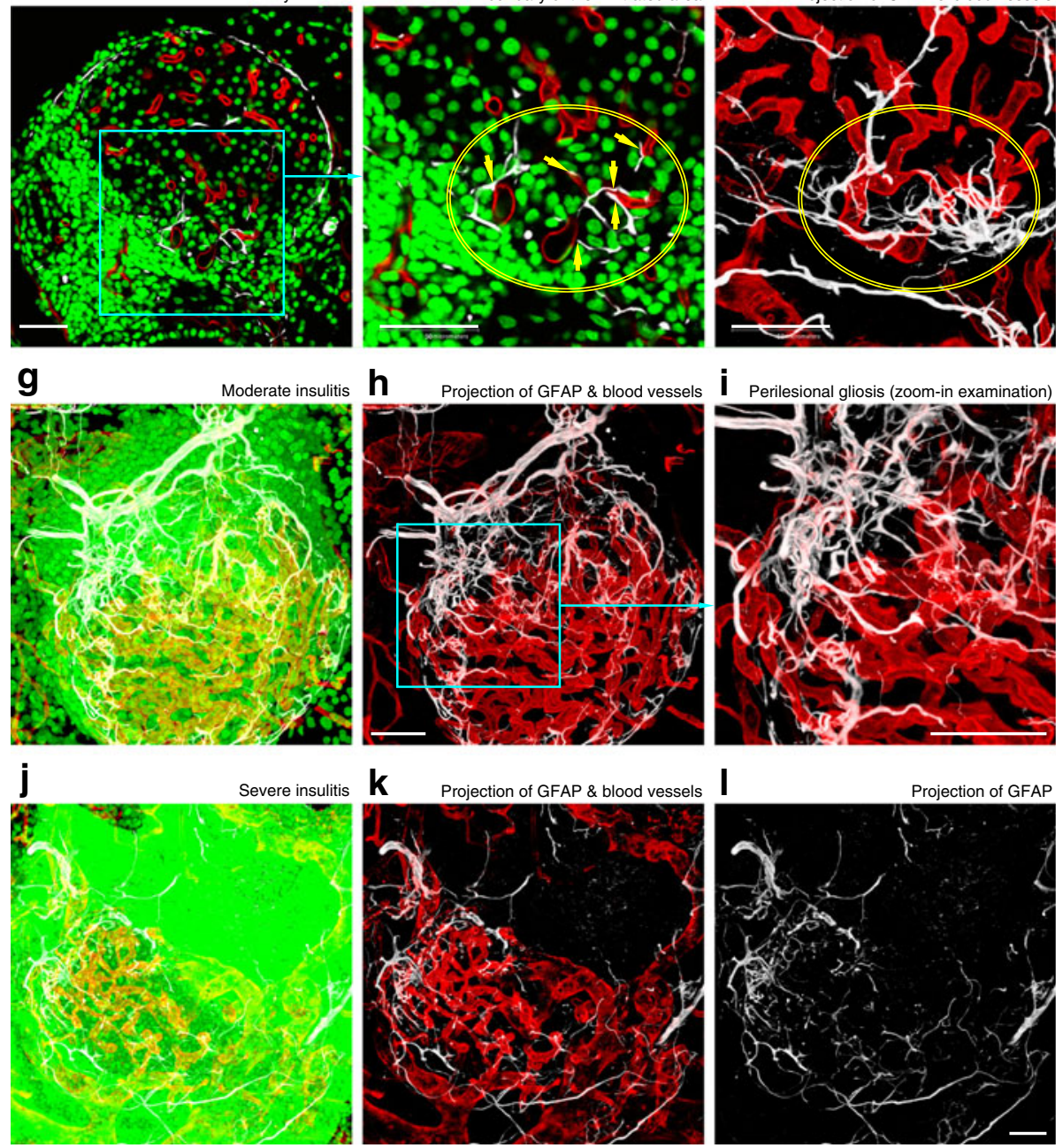

crosstalk, to present the image data with high fidelity (note: the ultraviolet channel was not used in this research due to limited penetration depth). Because two of the three channels were designated for observing the lesion (nuclear staining) and vasculature (vessel painting), only one was left to identify the marker of interest, either GFAP or NG2. Thus, we chose to characterise the Schwann cells and pericytes individually, but did not rule out their potential interaction or association with other network elements such as the nerves in the perilesional region. In fact, we used the same mouse model and imaging approach to reveal the perilesional remodelling of sympathetic nerves in insulitis [28], suggesting the generic plasticity of the pancreatic/islet neural tissues in response to injury. 
4 Fig. 5 Remodelling of the Schwann cell network in insulitis in NOD mice. (a-c) Gross view of gliosis in early insulitis in NOD mice. (a) Lymphocytic accumulation between the duct and the islet (5 $\mu \mathrm{m}$ optical section); white, GFAP; red, blood vessels; green, nuclei; scale bar, $100 \mu \mathrm{m}$. In early insulitis, lymphocytes typically migrate from the ductal area into the peri-islet domain against the mantle (yellow arrows). Blue arrows indicate the paths of the Schwann cell plexi coupled with those of the vasculature. (b,c) In-depth projections of the gliosis surrounding the area with inflammation. The influenced area is highlighted by vascular damage (asterisk shows area with loss of vessel painting signals) and a prominent increase in the GFAP content (blue circle) compared with the normal islet (Fig. 1c). Note that the staining background is elevated around the duct (double dagger). This is likely due to the attachment of apoptotic debris. Projection depth: $60 \mu \mathrm{m}$. (d-f) Close-up view of the perilesional and perivascular gliosis in early insulitis in NOD mice. (d) 2D Micrograph of the peri-islet lymphocytic infiltration (a still image of ESM Video 4). The area of the blue box in (d) is enlarged in (e) and projected in panel (f) (depth: $30 \mu \mathrm{m})$ to reveal the perivascular contacts between $\mathrm{GFAP}^{+}$fibres and capillaries (arrows) at the boundary of the infiltrated area (squashed circle). Short and prominent Schwann cell processes were seen at the front of the lymphocytic infiltration with abundant contacts between the processes and capillaries. The normal domain is at the upper right section of (d), in which the morphologies of the Schwann cell sheath and blood vessels remain similar to those of the normal islet. Scale bar: $50 \mu \mathrm{m}$ in all three panels. (g-i) Perilesional and perivascular gliosis in moderate insulitis in NOD mice. (g) Gross view of a remodelled Schwann cell network due to peri-islet lymphocytic infiltration. $(\mathbf{h}, \mathbf{i})$ Images at the infiltration boundary reveal intimate association between the Schwann cell processes and capillaries; scale bar, $50 \mu \mathrm{m}$; projection depth, $100.5 \mu \mathrm{m}$. In-depth visualisation of this islet was recorded in the second part of ESM Video 4. (j-l) Destruction of the Schwann cell network in severe insulitis in NOD mice. The in-depth projections show the GFAP, capillary and nuclear signals of a critically damaged islet. Remains of the dismantled Schwann cell network can still be seen in contact with the damaged and swelling vasculature; scale bar, $50 \mu \mathrm{m}$; projection depth, $60 \mu \mathrm{m}$

In summary, we characterised the plasticity of Schwann cells and pericytes in response to islet injury in experimental diabetes. Before this research, Schwann cells and pericytes had been overlooked with regard to their dynamic roles in the lesion during the progression of autoimmune diabetes. This is in part due to the difficulty in observing the islet tissue networks in a space continuum. We used a recently developed 3D imaging technique to resolve the spatial distribution of the tissue networks for qualitative and quantitative analyses of the two cell types to identify their reactive cellular responses in islet injury. Because several classes of molecular inducers and suppressors of glial scar have been recognised through studies on CNS injuries, future studies on manipulation of gliosis in experimental diabetes will further benefit our understanding of the

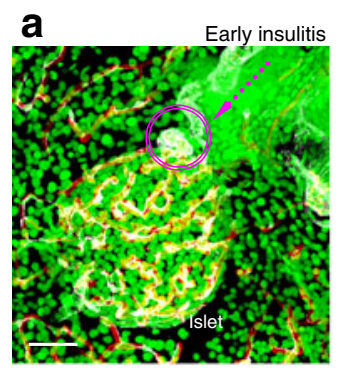

b
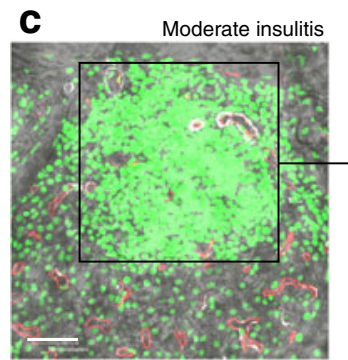

e
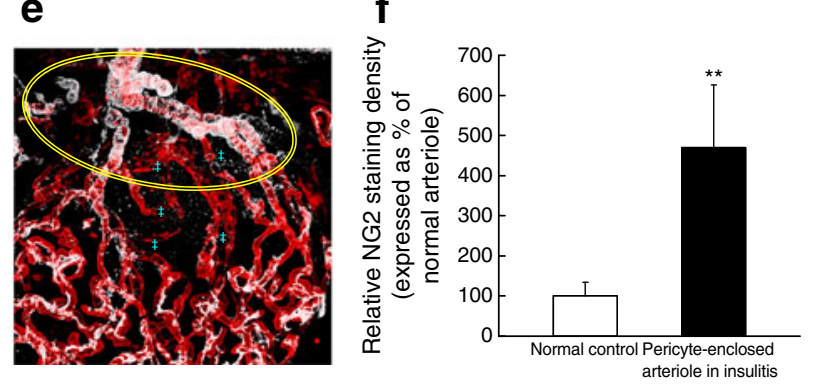

Fig. 6 Pericyte remodelling around the feeding arteriole in insulitis in NOD mice. $(\mathbf{a}, \mathbf{b})$ Pericyte aggregation at the feeding arteriole in early insulitis. Lymphocyte infiltration arrived at the islet pole (dotted arrow) leading to pericyte aggregation (circles) and an increase in NG2 staining density around the feeding arteriole; white, NG2; red, blood vessels; green, nuclei; scale bar, $50 \mu \mathrm{m}$; projection depth, $42 \mu \mathrm{m}$ and $61.5 \mu \mathrm{m}$ for (a) and (b), respectively. (c-f) Intralesional pericyte aggregation on the walls of the feeding arteriole in moderate insulitis. (c) Overlay of transmitted light and fluorescence image. (d) Zoom-in examination of the area with inflammation; white, NG2; red, blood vessels; green, nuclei; scale bar, $50 \mu \mathrm{m}$ in both (c) and (d). (e) Projection of pericytes and blood vessels (depth, $75 \mu \mathrm{m}$ ). Panels (c) and (e) were derived from the same view. At this stage, lymphocytes had infiltrated the islet core, yet pericyte aggregation on the feeding arteriole remained prominent (arrows in [d]) with the processes enclosing the vessel walls (squashed circle in [e]). On the other hand, loss of capillaries and their associated pericytes was seen as the lesion progressed toward the islet core (double daggers in $[\mathbf{e}]$ ). Quantification showed that the NG2 staining density was substantially higher on the feeding arteriole in insulitis than on the normal control. NG2 signal extraction and quantification are described in the "Methods" section. Data were derived from the normal $(n=3)$ and diseased NOD mice in early/ moderate insulitis $(n=5)$ and presented as means \pm SD. ${ }^{* *} p<0.01$ vs control

cellular components in the progression of islet lesions in type 1 diabetes. 
Acknowledgements We thank Ann-Shyn Chiang at the Brain Research Center, National Tsing Hua University for technical support in confocal imaging.

Funding This work was supported in part by grants from the Taiwan National Health Research Institutes and National Science Council to SCT.

Duality of interest The authors declare that there is no duality of interest associated with this manuscript.

Contribution statement All authors contributed to the experimental conception and design. YCC, CTH, SJP and YYF contributed to data acquisition, analysis and interpretation of data, and revised the manuscript critically for intellectual content. SCT directed the imaging project and contributed to the analysis and interpretation of data and the writing of the paper. All the authors approved the final version of the paper.

\section{References}

1. Woods SC, Porte D Jr (1974) Neural control of the endocrine pancreas. Physiol Rev 54:596-619

2. Donev SR (1984) Ultrastructural evidence for the presence of a glial sheath investing the islets of Langerhans in the pancreas of mammals. Cell Tissue Res 237:343-348

3. Sunami E, Kanazawa H, Hashizume H, Takeda M, Hatakeyama K, Ushiki T (2001) Morphological characteristics of Schwann cells in the islets of Langerhans of the murine pancreas. Arch Histol Cytol 64:191-201

4. Hayden MR, Karuparthi PR, Habibi J et al (2008) Ultrastructure of islet microcirculation, pericytes and the islet exocrine interface in the HIP rat model of diabetes. Exp Biol Med (Maywood) 233:1109-1123

5. Richards OC, Raines SM, Attie AD (2010) The role of blood vessels, endothelial cells, and vascular pericytes in insulin secretion and peripheral insulin action. Endocr Rev 31:343-363

6. Fawcett JW, Asher RA (1999) The glial scar and central nervous system repair. Brain Res Bull 49:377-391

7. Pekny M, Nilsson M (2005) Astrocyte activation and reactive gliosis. Glia 50:427-434

8. Goritz C, Dias DO, Tomilin N, Barbacid M, Shupliakov O, Frisen J (2011) A pericyte origin of spinal cord scar tissue. Science 333:238-242

9. von Boyen GB, Steinkamp M, Reinshagen M, Schafer KH, Adler G, Kirsch J (2004) Proinflammatory cytokines increase glial fibrillary acidic protein expression in enteric glia. Gut 53:222-228

10. Cornet A, Savidge TC, Cabarrocas J et al (2001) Enterocolitis induced by autoimmune targeting of enteric glial cells: a possible mechanism in Crohn's disease? Proc Natl Acad Sci U S A 98:1330613311

11. Costantini TW, Bansal V, Krzyzaniak M et al (2010) Vagal nerve stimulation protects against burn-induced intestinal injury through activation of enteric glia cells. Am J Physiol Gastrointest Liver Physiol 299:G1308-G1318

12. Vasina V, Barbara G, Talamonti L et al (2006) Enteric neuroplasticity evoked by inflammation. Auton Neurosci 126127:264-272

13. Winer S, Tsui H, Lau A et al (2003) Autoimmune islet destruction in spontaneous type 1 diabetes is not beta-cell exclusive. Nat Med 9:198-205

14. Tsui H, Winer $\mathrm{S}$, Chan $\mathrm{Y}$ et al (2008) Islet glia, neurons, and beta cells. Ann N Y Acad Sci 1150:32-42
15. Tsui H, Chan Y, Tang L et al (2008) Targeting of pancreatic glia in type 1 diabetes. Diabetes 57:918-928

16. Yantha J, Tsui H, Winer S et al (2010) Unexpected acceleration of type 1 diabetes by transgenic expression of B7-H1 in NOD mouse peri-islet glia. Diabetes 59:2588-2596

17. Teitelman G, Guz Y, Ivkovic S, Ehrlich M (1998) Islet injury induces neurotrophin expression in pancreatic cells and reactive gliosis of peri-islet Schwann cells. J Neurobiol 34:304-318

18. Nakamura M, Kitamura H, Konishi S et al (1995) The endocrine pancreas of spontaneously diabetic $\mathrm{db} / \mathrm{db}$ mice: microangiopathy as revealed by transmission electron microscopy. Diabetes Res Clin Pract 30:89-100

19. Hayden MR, Karuparthi PR, Habibi J et al (2007) Ultrastructural islet study of early fibrosis in the Ren2 rat model of hypertension. Emerging role of the islet pancreatic pericyte-stellate cell. JOP 8:725-738

20. Papaccio G, Latronico MV, Pisanti FA, Federlin K, Linn T (1998) Adhesion molecules and microvascular changes in the nonobese diabetic (NOD) mouse pancreas. An NO-inhibitor (L-NAME) is unable to block adhesion inflammation-induced activation. Autoimmunity 27:65-77

21. Denis MC, Mahmood U, Benoist C, Mathis D, Weissleder R (2004) Imaging inflammation of the pancreatic islets in type 1 diabetes. Proc Natl Acad Sci U S A 101:12634-12639

22. Tuchin VV, Wang RK, Yeh AT (2008) Optical clearing of tissues and cells. J Biomed Opt 13:021101

23. Fu YY, Lin CW, Enikolopov G, Sibley E, Chiang AS, Tang SC (2009) Microtome-free 3-dimensional confocal imaging method for visualization of mouse intestine with subcellular-level resolution. Gastroenterology 137:453-465

24. Fu YY, Tang SC (2010) At the movies: 3-dimensional technology and gastrointestinal histology. Gastroenterology 139:1100-1105

25. Hama H, Kurokawa H, Kawano H et al (2011) Scale: a chemical approach for fluorescence imaging and reconstruction of transparent mouse brain. Nat Neurosci 14:1481-1488

26. Liu YA, Chung YC, Pan ST et al (2013) 3-D imaging, illustration, and quantitation of enteric glial network in transparent human colon mucosa. Neurogastroenterol Motil 25:e324-e338

27. Fu YY, Lu CH, Lin CW et al (2010) Three-dimensional optical method for integrated visualization of mouse islet microstructure and vascular network with subcellular-level resolution. J Biomed Opt 15:046018

28. Chiu YC, Hua TE, Fu YY, Pasricha PJ, Tang SC (2012) 3-D imaging and illustration of the perfusive mouse islet sympathetic innervation and its remodelling in injury. Diabetologia 55:3252-3261

29. Ahren B (2012) Islet nerves in focus - defining their neurobiological and clinical role. Diabetologia 55:3152-3154

30. Fu YY, Peng SJ, Lin HY, Pasricha PJ, Tang SC (2013) 3-D imaging and illustration of mouse intestinal neurovascular complex. Am J Physiol Gastrointest Liver Physiol 304:G1-G11

31. Liu YA, Chung YC, Pan ST et al (2012) 3-D illustration of network orientations of interstitial cells of Cajal subgroups in human colon as revealed by deep-tissue imaging with optical clearing. Am J Physiol Gastrointest Liver Physiol 302:G1099-G1110

32. Liu YA, Chen Y, Chiang AS, Peng SJ, Pasricha PJ, Tang SC (2011) Optical clearing improves the imaging depth and signal-to-noise ratio for digital analysis and three-dimensional projection of the human enteric nervous system. Neurogastroenterol Motil 23:e446-e457

33. Bergers G, Song S (2005) The role of pericytes in blood-vessel formation and maintenance. Neuro Oncol 7:452-464

34. Ravnic DJ, Jiang X, Wolloscheck T et al (2005) Vessel painting of the microcirculation using fluorescent lipophilic tracers. Microvasc Res 70:90-96

35. Fu YY, Tang SC (2010) Optical clearing facilitates integrated 3D visualization of mouse ileal microstructure and vascular network with high definition. Microvasc Res 80:512-521 
36. Taylor-Fishwick DA, Bowman A, Korngiebel-Rosique M, Vinik AI (2008) Pancreatic islet immunoreactivity to the Reg protein INGAP. J Histochem Cytochem 56:183-191

37. Carl Zeiss Microimaging GmbH (2009) Visualizing the architecture of cells and tissues. Brochures for laser scanning microscopy. Available from http://microscopy.zeiss.com/microscopy/en_gb/ downloads/brochure-downloads.html?catalog=biowhitepaper. Accessed 14 June 2013

38. Sandler S, Jansson L (1985) Vascular permeability of pancreatic islets after administration of streptozotocin. Virchows Arch A Pathol Anat Histopathol 407:359-367

39. Papaccio G (1993) Insulitis and islet microvasculature in type 1 diabetes. Histol Histopathol 8:751-759

40. Beppu H, Maruta K, Kurner T, Kolb H (1987) Diabetogenic action of streptozotocin: essential role of membrane permeability. Acta Endocrinol (Copenh) 114:90-95
41. Enghofer M, Usadel KH, Beck O, Kusterer K (1997) Superoxide dismutase reduces islet microvascular injury induced by streptozotocin in the rat. Am J Physiol 273:E376-E382

42. Papaccio G, Baccari GC, Strate C, Linn T (1994) Pancreatic duct inflammatory infiltration in the nonobese diabetic (NOD) mouse. J Anat 185:465-470

43. Tsui H, Winer S, Jakowsky G, Dosch HM (2003) Neuronal elements in the pathogenesis of type 1 diabetes. Rev Endocr Metab Disord 4:301-310

44. Meyerzu Horste G, Hu W, Hartung HP, Lehmann HC, Kieseier BC (2008) The immunocompetence of Schwann cells. Muscle Nerve 37:3-13

45. Wekerle H, Schwab M, Linington C, Meyermann R (1986) Antigen presentation in the peripheral nervous system: Schwann cells present endogenous myelin autoantigens to lymphocytes. Eur J Immunol 16:1551-1557 